\title{
Stacking interactions and the twist of DNA
}

Valentino R. Cooper ${ }^{1, *}$, Timo Thonhauser ${ }^{l}$, Aaron Puzder ${ }^{1, a}$, Elsebeth Schröder $^{2}$, Bengt I. Lundqvist ${ }^{2,3}$, and David C. Langreth ${ }^{1}$

${ }^{1}$ Department of Physics and Astronomy, Rutgers University, Piscataway, New Jersey 08854-8019, USA

${ }^{2}$ Department of Applied Physics, Chalmers University of Technology, SE-412 96 Göteborg, Sweden

${ }^{3}$ Department of Physics, Technical University of Denmark, DK-2800 Lyngby, Denmark

${ }^{*}$ To whom correspondence should be addressed; E-mail: vcooper@physics.rutgers.edu.

\section{Supporting Information - full version of references}

(27) Olson, W. K.; Bansal, M.; Burley, S. K.; Dickerson, R. E.; Gerstein, M.; Harvey, S. C.; Heinemann, U.; Lu, X.-J.; Neidle, S.; Shakked, Z.; Sklenar, H.; Suzuki, M.; Tung, C.-S.; Westhof, E.; Wolberger, C.; Berman, H. M. J. Mol. Biol. 2001, 313, 229.

(32) Gonze, X.; Beuken, J.-M.; Caracas, R.; Detraux, F.; Fuchs, M.; Rignanese, G.-M.; Sindic, L.; Verstraete, M.; Zerah, G.; Jollet, F.; Torrent, M.; Roy, A.; Mikami, M.; Ghosez, P.; Raty, J.-Y.; Allan, D. Comp. Mat. Sci. 2002, 25, 478.

a Present Address: Lawrence Livermore National Lab, 7000 East Avenue, L-415, Livermore, CA 94550 
Supporting Information Table 1. DNA base pair interaction energy at preferred helical twist angle and base pair separation distance. DNA notation follows that of the Nucleic Acid Database. ${ }^{1,2}$

\begin{tabular}{|c|c|c|c|}
\hline DNA Base Pair & $\begin{array}{l}\text { Interaction Energy } \\
(\mathrm{kcal} / \mathrm{mol})\end{array}$ & $\begin{array}{c}\text { Preferred Helical } \\
\text { Twist Angle }\left(^{\circ}\right)\end{array}$ & $\begin{array}{c}\text { Preferred Dimer } \\
\text { Separation Distance }(\AA)\end{array}$ \\
\hline$\overline{\mathrm{AA}: \mathrm{TT}}$ & -15.2 & 40.6 & 3.51 \\
\hline AA:UU & -14.8 & 45.5 & 3.46 \\
\hline AT:AT & -14.0 & 17.6 & 3.60 \\
\hline AU:AU & -13.5 & 50.3 & 3.60 \\
\hline TA:TA & -15.1 & 38.2 & 3.46 \\
\hline UA:UA & -15.1 & 38.2 & 3.45 \\
\hline GG:CC & -13.4 & 47.9 & 3.51 \\
\hline $\mathrm{GC}: \mathrm{GC}$ & -17.0 & 21.2 & 3.53 \\
\hline $\mathrm{CG}: \mathrm{CG}$ & -18.2 & 33.3 & 3.45 \\
\hline AG:CT & -15.2 & 40.6 & 3.53 \\
\hline $\mathrm{AG}: \mathrm{CU}$ & -15.5 & 40.6 & 3.48 \\
\hline GA:TC & -13.9 & 38.2 & 3.52 \\
\hline GA:UC & -13.7 & 37.0 & 3.53 \\
\hline AC:GT & -14.5 & 21.2 & 3.58 \\
\hline $\mathrm{AC}: \mathrm{GU}$ & -13.8 & 38.2 & 3.54 \\
\hline CA:TG & -16.3 & 39.4 & 3.44 \\
\hline CA:UG & -16.3 & 40.6 & 3.44 \\
\hline Average $^{b}$ : & $-15.3 \pm 1.5$ & $33.8 \pm 10.2$ & $3.51 \pm 0.05$ \\
\hline
\end{tabular}

b Averages do not include base pair steps with uracil containing nucleobases. 
Supporting Information Table 2. DNA average Twist parameters for DNA dinucleobase steps obtained from x-ray crystallography analysis and theoretical modeling of DNA steps without a sugar-phosphate backbone. Columns 2-4 summarize the analysis by El Hassan et al., ${ }^{3}$ Gorin et al. ${ }^{4}$ and Olson et al. ${ }^{5}$ of Twists of steps of DNA along with their standard deviations. Column 5 shows the corresponding vdW-DF prediction for the same steps of an isolated dimer of two base pairs (without backbone). For entries marked (flat), the curves in Figures 3 and 4, were sufficiently flat in the region surrounding their minima that it was deemed unlikely that the stacking forces play much of a role in determining the Twist. Column 6 contains force-field predictions for the corresponding dimers. ${ }^{6,7}$ As opposed to column 5 entries where both the Twist and Rise were optimized, the column 6 entries were made with a fixed rise of $3.36 \AA$. There are of course no standard deviations associated with columns 5 and 6, but, as clear from Figures 3 and 4 and the discussion in the text, energy contributions from other sources not included in the calculations easily add uncertainty to the calculated numbers at least as large as the experimental standard deviations (much larger in the cases labeled as flat).

\begin{tabular}{lccccc}
\hline \hline DNA Base Pair & El Hassan et al. $^{3}$ & Gorin et al. $^{4}$ & Olson et al. $^{5}$ & vdW-DF & Force-Field $^{6,7}$ \\
\hline \hline AA:TT & $36 \pm 3$ & $36 \pm 3$ & $35 \pm 4$ & 41 & 37 \\
AC:GT & $33 \pm 4$ & $36 \pm 4$ & $32 \pm 4$ & 22 (flat) & $<30$ \\
AG:CT & $36 \pm 4$ & $31 \pm 5$ & $32 \pm 5$ & 41 & 37 \\
AT:AT & $32 \pm 3$ & $33 \pm 4$ & $29 \pm 5$ & 18 (flat) & $<30$ \\
CA:TG & $37 \pm 10$ & $37 \pm 10$ & $37 \pm 7$ & 39 & 35 \\
CG:CG & $35 \pm 5$ & $31 \pm 5$ & $36 \pm 6$ & 33 & 33 \\
GA:TC & $38 \pm 4$ & $39 \pm 3$ & $36 \pm 4$ & 38 & 35 \\
GC:GC & $37 \pm 4$ & $38 \pm 4$ & $34 \pm 5$ & 21 & $<30$ \\
GG:CC & $32 \pm 4$ & $33 \pm 3$ & $33 \pm 5$ & 48 (flat) & $>42$ \\
TA:TA & $31 \pm 7$ & $40 \pm 4$ & $38 \pm 6$ & 38 & 35 \\
\hline \hline
\end{tabular}




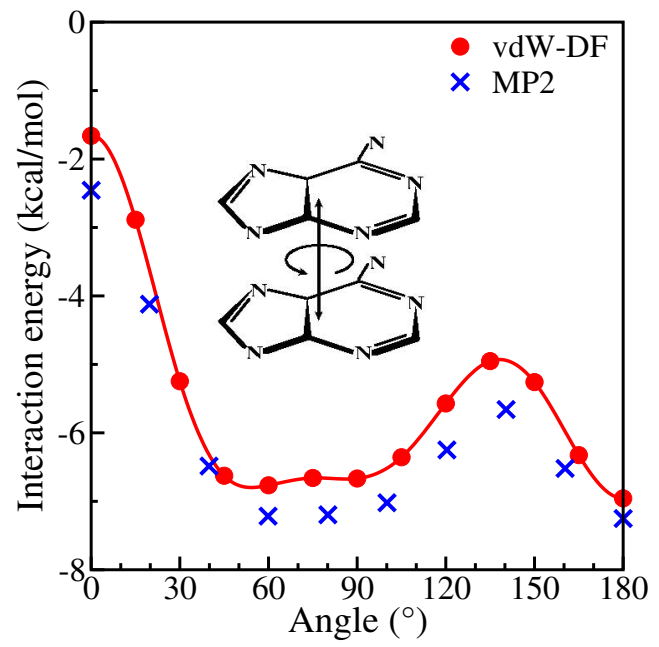

Supporting Information Figure 1. Comparison between vdW-DF (red dots) and previous MP2 calculations by Elstner et al. ${ }^{8}$ (blue crosses) for the stacking interaction energy as a function of rotation angle for the adenine-adenine dimer. These results highlight the good agreement between vdW-DF and MP2 calculations. Similar calculations have been made for a number of other nucleobase pairs. The above is typical of the agreement obtained. 


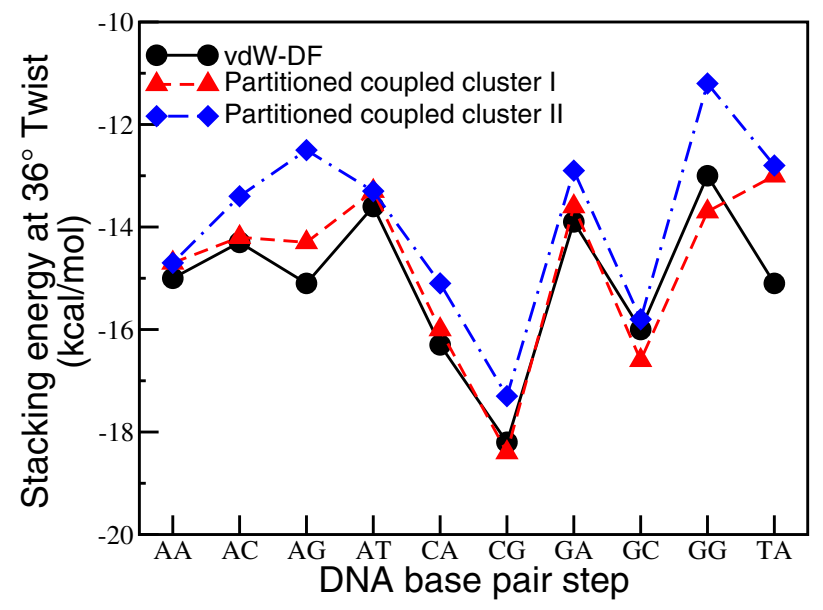

Supporting Information Figure 2. Comparison between vdW-DF and the calculations of Šponer et al. ${ }^{6}$ at $36^{\circ}$ Twist. In Ref. 6 a standard high-level CCSD(T)-based technique extrapolated to the complete basis set limit is applied to partitioned subsystems that are small enough to make the calculations feasible and combines the results from the several partitions (Partitioned coupled cluster I). The authors estimate the error and add the estimate using MP2 with a smaller basis set (Partitioned coupled cluster II). The partitioning approximation was not used for the vdW-DF calculations. Labels refer to the left strand only. 


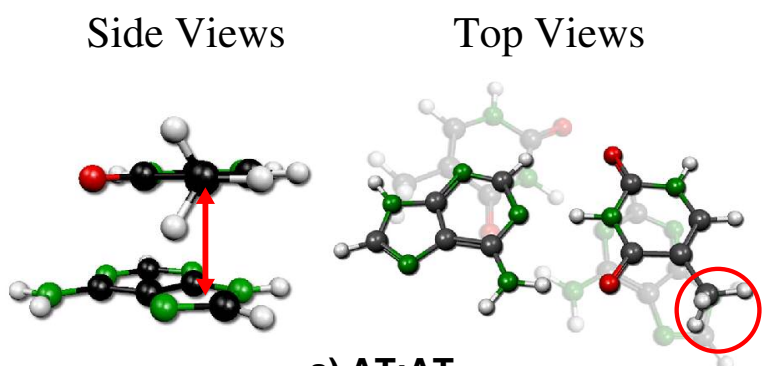

a) AT:AT
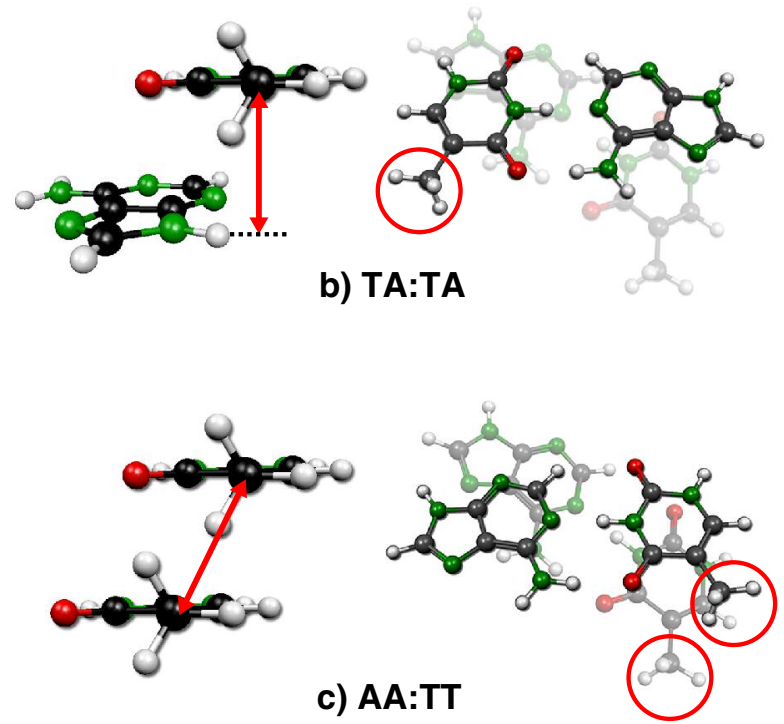

Supporting Information Figure 3. Side (left) and top (right) views of the thymine contact points at Twists of $36^{\circ}$ for three representative stacked nucleobase pairs a) AT:AT, b) TA:TA and c) AA:TT. In both a) and c) methyl-nucleobase and methyl-methyl interactions result in significant enhancement of the stacking energy relative to the uracil containing counterparts. TA:TA shows no appreciable difference as the thymine methyl group is rotated away from the adjacent nucleobase. 


\section{References}

(1) Olson, W. K. et al. J. Mol. Biol. 2001, 313, 229.

(2) Berman, H. M.; Olson, W. K.; Beveridge, D. L.; Westbrook, J.; Gelbin, A.; Demeny, T.; Hsieh, S.-H.; Srinivasan, A. R.; Schneider, B. Biophys. J. 1992, 63, 751 (http://ndbserver.rutgers.edu).

(3) El Hassan, M. A.; Calladine, C. R. Phil. Trans.: Ser. A 1997, 355, 43.

(4) Gorin, A. A.; Zhurkin, V. B.; Olson, W. K. J. Mol. Biol. 1995, 247, 34.

(5) Olson, W. K.; Gorin, A. A.; Lu, X.-J.; Hock, L. M.; Zhurkin, V. B. PNAS 1998, 95, 11163.

(6) Šponer, J.; Jurecka, P.; Marchan, I.; Luque, F. J.; Orozoco, M.; Hobza, P. Chem. Eur. J. 2006, 12, 2854.

(7) Cornell, W. D.; $\quad$ Cieplak, P.; $\quad$ Bayly, C. I.; $\quad$ Gould, I. R.; $\quad$ Merz, K. M.; $\quad$ Ferguson, D. M.; Spellmeyer, D. C.; Fox, T.; Caldwell, J. W.; Kollman, P. A. J. Am. Chem. Soc. 1995, 117, 5179.

(8) Elstner, M.; Hobza, P.; Frauenheim, T.; Suhai, S.; Kaxiras, E. J. Chem. Phys. 2001, 114, 5150. 